\title{
Status of the brain antioxidant system at different growing periods after prenatal stress and $\mathrm{N}$-acetyl cysteine administration
}

\author{
Liegelin Kavitha Bernhardt ${ }^{1}$, Sampath Madhyastha ${ }^{2}$, Lakshminarayana Bairy ${ }^{3}$, Anoop Kishore ${ }^{4}$ \\ ${ }^{1}$ Department of Physiology, Melaka Manipal Medical College, Manipal University, Manipal, India, ${ }^{2}$ Department of Anatomy, \\ Faculty of Medicine, Kuwait University, Kuwait, ${ }^{3}$ Department of Pharmacology, Kasturba Medical College, Manipal University, \\ Manipal, India, ${ }^{4}$ Department of Pharmacology, MCOPS, Manipal University, Manipal, India
}

\begin{abstract}
Prenatal stress-induced neurobehavioral deficits observed in offspring are multifactorial, including oxidative stress in the developing brain. The time by which the developing brain acquires self-defense against oxidative stress is not clear. Hence in the present study we aimed to evaluate the brain antioxidant status during different developing periods. Further the study also evaluates the role of the glutathione precursor, $N$-acetyl cysteine (NAC) on the brain antioxidant status. Pregnant rats were subjected to restraint stress during an early or late gestational period. Another set of rats received NAC during the entire gestational period along with early or late gestational stress. The study parameters included several antioxidant studies directly from rat brain homogenate on postnatal day 24 or 48 . Early or late gestational stress has caused severe oxidative stress in the developing brain on postnatal day 24 in all the parameters studied. However, brain reduced glutathione (GSH), superoxide dismutase (SOD) and total antioxidant activity (TAO) were not affected by either early or late gestational stress on postnatal day 48, but the brain malondialdehyde (MDA) level remained high and brain glutathione reductase (GSS-Rd) level remained low on postnatal day 48. Prenatal NAC treatment has reversed the oxidative damage in all the parameters on postnatal day 24 and also the brain MDA level and GSS-Rd level on postnatal day 48. This study confirms that the growing brain acquires antioxidant capacity over time but during early postnatal development it is vulnerable to oxidative stress and related neurological consequences. $\mathrm{N}$-acetyl cysteine treatment during the prenatal period as an antioxidant supplement exerted a beneficiary effect in this study. Hence glutathione supplement in the nutritional source would be an idealistic approach to prenatal stress-induced neurological comorbidities in children.
\end{abstract}

Key words: brain antioxidant system, brain oxidative stress, $\mathrm{N}$-acetyl cysteine, prenatal stress.

\section{Introduction}

It is well known that the prenatal stress adversely affects the brain development with neuronal loss and behavioral dysfunction. Among the various mecha- nisms involved in the developmental toxicity, the altered cellular homeostasis by oxidative damage in the developing brain has gained much attention in recent years. The adult brain is known to be well equipped with defense and repair mechanisms to combat

\section{Communicating author:}

Dr. Sampath Madhyastha, PhD, Associate Professor, Department of Anatomy, Faculty of Medicine, Kuwait University, Kuwait, phone: 0096594130871 , e-mail: sampath.m@hsc.edu.kw 
the oxidative stress, but existence of such defensive mechanisms in the developing brain is obscure [14]. Oxidative stress occurs when free radicals are generated more rapidly than they are buffered by the cellular defense mechanisms [4]. Maintenance of a balance between reactive oxygen species (ROS) production and antioxidant defense is very essential for normal development of organisms. Prenatal stress-induced suppression of neuronal proliferation involving brain antioxidant systems was reported by Kawamura et al. [16]. Prenatal stress-induced oxidative damage to mitochondrial DNA and damage to hippocampal neurons in neonates is reported by Zhu et al. [43]. Hence the cellular damage in the developing brain could be attributed to overproduction of antioxidants.

Administration of an antioxidant which crosses both the placental and blood brain barrier may be helpful in providing the antioxidant defense system in the growing brain. $N$-acetyl cysteine (NAC), a thiol compound having the formula $\mathrm{C} 5 \mathrm{H} 9 \mathrm{O} 3 \mathrm{~S}$ is a very good source of sulfhydryl $(\mathrm{SH})$ groups that stimulate the reduced glutathione (GSH) synthesis, help detoxification and directly bring about free radical scavenging [44]. Among the various studies conducted on neuroprotective interventions aiming at ameliorating brain injury, only few have been proved safe for clinical use. $N$-acetyl cysteine is one such antioxidant, which is proved to be safe during pregnancy. The efficacy of NAC as a neuroprotector is largely due to its ability to stimulate the reduced GSH synthesis by acting as a cysteine donor [7], cross the bloodbrain barrier [12] and boost brain GSH levels during periods of increased oxidative stress [12,19]. Previous studies have demonstrated that NAC has reduced the oxidative stress in rat brains produced by lead exposure [29], limited maternal lipopolysaccharide (LPS) induced oxidative stress and increased lipid peroxidation in the placenta that led to preterm labor [33], prevented birth-linked oxidative stress in neonates, when pre administered to dams [37]. Lante et al. [19] found that prenatal exposure to lipopolysaccharide brought about oxidative stress in fetal hippocampus while pretreatment with NAC protected the offspring from impairment of the spatial memory and synaptic plasticity [19]. $\mathrm{N}$-acetyl cysteine has been proven to protect the brain against oxidative stress and neurodevelopmental damage associated with prenatal infection [30]. Hence in the present study, NAC, a wellknown antioxidant was considered as a therapeutic strategy to combat the prenatal stress-induced possible oxidative damage in the developing brain.

\section{Material and methods \\ Animals}

Healthy, in-house bred female and male rats of the albino Wistar strain (3-4 months of age) were obtained for the study. The rats were maintained in 12 hours light and dark cycle in temperature- and humidity-controlled environment. The rats were fed with standard food pellet and water ad libitum. The polypropylene cage with paddy husk as bedding materials was used for housing the rats. Breeding and maintenance of the animals were done as per the guidelines of the Government of India for use of laboratory animals and also ARRIVE guidelines. The Institutional Animal Ethics Committee approval was obtained before the conduct of the study and care was taken to handle the rats in a humane manner.

\section{Animal breeding}

Female rats were allowed to mate with one fertile sexually active male for four hours per day (separate male rats for each group). At the end of four hours, female rats were separated and vaginal smears taken to detect the presence of sperm for the confirmation of pregnancy and the rats were designated as day 0 of pregnancy for further counting the days. The pregnant rats were housed individually in separate cages with a proper label indicating the day of conception and randomly allocated into six groups of eight each. One male and one female pup from each mother (a total of 16 pups in each group) were considered for antioxidant studies.

\section{Induction of prenatal stress}

The pregnant rats were stressed (restraint stress) using a wire mesh restrainer [24] three times daily for $45 \mathrm{~min}$. The wire mesh restrainer had a wooden base and stainless steel wire mesh restrainer hinged to the base. A pad lock and latch helped to secure the rat in the restrainer. The restrainer with dimensions of $11 \mathrm{~cm}(\mathrm{~L}) \times 6 \mathrm{~cm}(\mathrm{~B}) \times 6 \mathrm{~cm}(\mathrm{H})$ was used for rats with gestation day 1 to 10 . The restrainer of $11 \mathrm{~cm} \mathrm{(L)} \times 8 \mathrm{~cm}(\mathrm{~B}) \times 8 \mathrm{~cm}(\mathrm{H})$ was used for rats with gestation day 11 to till delivery. This type of restrainer will only restrict the animal movement without any pain, discomfort or suffocation. 


\section{Animal groups and treatment}

Group 1 (Control): The pups belonging to the pregnant rats who received only normal saline in a dose of $10 \mathrm{ml} / \mathrm{kg}$ body weight (intraperitoneal) throughout pregnancy.

Group 2 (NAC): The pups belonging to the pregnant rats who received NAC alone in a dose of $10 \mathrm{mg} /$ kg body weight (i.p.) throughout pregnancy.

Group 3 (EGS): The pups belonging to the pregnant rats who received restrain stress from gestation day 1 to 10 and normal saline throughout pregnancy.

Group 4 (LGS): The pups belonging to the pregnant rats who received restrain stress from gestation day 11 to delivery and normal saline throughout pregnancy.

Group 5 (EGS+NAC): The pups belonging to the pregnant rats who received restrain stress from gestation day 1 to 10 and NAC (10 mg/kg body weight, i.p.) throughout pregnancy.

Group 6 (LGS+NAC): The pups belonging to the pregnant rats who received restrain stress from gestation day 11 to delivery and NAC (10 mg/kg body weight, i.p.) throughout pregnancy.

All the mothers delivered at term (21-24 ${ }^{\text {th }}$ day of gestation). The offspring of the all groups were raised by their biological mothers until weaning $\left(21^{\text {st }}\right.$ day after birth).

\section{$\mathrm{N}$-acetyl cysteine treatment}

The dose of NAC was selected based on an earlier study by Basyigit et al. [1] where a $10 \mathrm{mg} / \mathrm{kg}$ dose (i.p.) was given continuously for 14 days to pregnant rats. The acute oral toxicity of NAC is low e.g. LD 50 $>10,000 \mathrm{mg} / \mathrm{kg}$ body weight in adult rats. In rats administered with $250 \mathrm{mg} \mathrm{NAC/kg}$ body weight per day for 20 weeks, all NAC-related effects observed are marginal. Hence in the present study, a $10 \mathrm{mg} / \mathrm{kg}$ dose of NAC dissolved in physiological saline was administered intraperitoneally.

For the present study, one male and one female pup from each mother were selected randomly. Pups were divided into two groups: the first group was sacrificed on postnatal day 24 (PND 24) and the other group was sacrificed on postnatal day 48 (PND 48) for antioxidant estimations; to assess both shortand long-term effects of prenatal stress.

We aimed at investigating the effect of prenatal stress during two important phases of life, which are childhood and adolescence. PND 24 in rats appears to correspond to the childhood period in humans.
This is the time period in rats when structural maturation of the brain takes place. A plateau in the volume of most brain structures has been found to occur during this period. PND 48 was selected so as to investigate whether the effects of early and late gestational stress last until the adolescent/early adult period. Many adolescent specific neurobehavioral alterations observed in humans are also seen in rats of comparable age. Adolescence is an age period around the time of sexual maturation when age-specific behavioral and psychopharmacological discontinuities are evident.

\section{Estimation of oxidative stress in brain}

Preparation of brain homogenate: Pups were weighed and sacrificed by decapitation after ether anesthesia. The whole brain was removed rapidly and rinsed with $0.1 \mathrm{M} / \mathrm{l}$ saline phosphate buffer $(\mathrm{pH} 7.4)$. Tissue was weighed and homogenized $(1: 10 \mathrm{w} / \mathrm{v})$ in $0.1 \mathrm{M} / \mathrm{I}$ saline phosphate buffer. The homogenate was centrifuged at $10000 \mathrm{~g}$ for $20 \mathrm{~min}$ at $4^{\circ} \mathrm{C}$ and aliquots of supernatant were separated and used for following biochemical estimations.

Estimation of total antioxidants: Brain total antioxidant capacity was assayed according to the method of Koracevic et al. [17]. The method is based on determination of the ability to eliminate added hydrogen peroxide. The remaining $\mathrm{H}_{2} \mathrm{O}_{2}$ is determined calorimetrically by an enzymatic reaction converting 3 , 5-dichloro-2-hydroxyl benzenesulfonate to a colored product that is measured at $532 \mathrm{~nm}$.

Estimation of superoxide dismutase activity: Superoxide dismutase (SOD) activity was determined by the method of Marklund et al. [25]. The reaction was performed in an mixture containing $5.6 \times 10^{-5} \mathrm{M}$ nitro blue tetrazolium (NBT), $1.17 \times 10^{-6} \mathrm{M}$ riboflavin, $1 \times 10^{-2} \mathrm{M}$ methionine in $0.05 \mathrm{M}$ potassium phosphate buffer, pH 7.8 with suitably diluted tissue homogenate in a total volume of $3 \mathrm{ml}$. Illumination of the solution was carried out in an aluminum lined foil box fitted with a 15v fluorescent lamp. The solution taken in a beaker was kept in the box and illuminated exactly for $10 \mathrm{~min}$. The control without the enzyme source was prepared. The absorbance was measured spectrophotometrically with a Systronic-117 UV-Visible spectrophotometer at $560 \mathrm{~nm}$. SOD activity was expressed as specific activity of the enzyme in units per $\mathrm{mg}$ protein (U/mg protein). Protein content was determined by the method of Lowry et al. [21]. 
Estimation of reduced glutathione: Glutathione in the brain was estimated according to the method described by Ellman [10]. $1 \mathrm{ml}$ supernatant was precipitated with $1 \mathrm{ml}$ of $4 \%$ sulfosalicylic acid and cold digested at $41^{\circ} \mathrm{C}$ for 1 hour. The samples were centrifuged at $1200 \mathrm{~g}$ for $15 \mathrm{~min}$ at $41^{\circ} \mathrm{C}$. To $1 \mathrm{ml}$ of this supernatant, $2.7 \mathrm{ml}$ of phosphate buffer $(0.1 \mathrm{~mol} / \mathrm{l}$, $\mathrm{pH} 8$ ) and $0.2 \mathrm{ml}$ of 5, 50-dithio-bis (2-nitrobenzoic acid) were added. The yellow color developed was read immediately at $412 \mathrm{~nm}$ by using the UV- 1700 spectrophotometer, Shimadzu, Japan. Results were calculated using a molar extinction coefficient of chromophore $\left(1.36 \times 10^{4} / \mathrm{mol} / / / \mathrm{cm}\right)$ and expressed as nmol per mg protein.

Estimation of glutathione reductase: The GSSG$\mathrm{Rd}$ activity was measured using the method originally described by Moron et al. [27]. The reaction mixture consisted of $1.6 \mathrm{ml}$ of $0.067 \mathrm{M}$ potassium phosphate buffer ( $\mathrm{pH} 6.6$ ), $0.12 \mathrm{ml}$ of $0.06 \% \mathrm{NADPH}, 0.12 \mathrm{ml}$ $1.15 \%$ GSSG, $0.1 \mathrm{ml}$ of enzyme source and water in a final volume of $2 \mathrm{ml}$. All mixtures and solutions were prepared at room temperature. Control cuvettes then received $180 \mu \mathrm{l}$ of deionized water while sample cuvettes received $60 \mu \mathrm{l}$ of deionized water and $120 \mu \mathrm{l}$ of GSSG solution. NADPH oxidation was followed for 5 min and was recorded using a Systronic-117 spectrophotometer. The reduction of GSSG to GSH was determined indirectly by the measurement of the consumption of NADPH, as demonstrated by a decrease in absorbance at $340 \mathrm{~nm}$ as a function of time. The enzyme activity was calculated using the extinction coefficient of chromophore $\left(1.36 \times 10^{4}\left(\mathrm{~mol} / \mathrm{l}^{-1} \mathrm{~cm}^{-1}\right)\right.$ and expressed as nmol NADPH oxidized/min/mg protein. Protein content was determined by the method of Lowry et al. [21] with bovine serum albumin as standard.

Estimation of lipid peroxidation: Measurement of lipid peroxidation was done by the method described by Ohkawa et al. [31]. Tissue lipid peroxidation was measured in whole-brain homogenate for $30 \mathrm{~min}$ at $37^{\circ} \mathrm{C}$. The incubation was interrupted by adding

Table I. Brain oxidant/antioxidant levels in rat brain at PND 24 and PND 48

\begin{tabular}{|c|c|c|c|c|c|c|c|c|c|c|c|}
\hline \multicolumn{12}{|c|}{ Brain oxidant/antioxidants (PND 24) } \\
\hline & & \multicolumn{2}{|c|}{$\begin{array}{l}\text { Total antioxidants } \\
\qquad(\mathrm{mmol} / \mathrm{l})\end{array}$} & \multicolumn{2}{|c|}{$\begin{array}{c}\text { Superoxide } \\
\text { dismutase } \\
\text { (U/mg protein) }\end{array}$} & \multicolumn{2}{|c|}{$\begin{array}{l}\text { Reduced } \\
\text { glutathione } \\
\text { (mg/gm } \\
\text { brain tissue) }\end{array}$} & \multicolumn{2}{|c|}{$\begin{array}{l}\text { Glutathione } \\
\text { reductase (nmol } \\
\text { NADPH oxidized/ } \\
\text { min/mg protein) }\end{array}$} & \multicolumn{2}{|c|}{$\begin{array}{l}\text { Lipid peroxidation } \\
\text { (ng/gm) }\end{array}$} \\
\hline \multirow[t]{2}{*}{ CON } & $\begin{array}{l}\text { Male } \\
(n=4)\end{array}$ & $\begin{array}{c}1.97 \pm \\
0.60\end{array}$ & \multirow{2}{*}{$\begin{array}{l}p=0.83 \\
t=0.22\end{array}$} & $\begin{array}{c}1.72 \pm \\
0.25\end{array}$ & \multirow{2}{*}{$\begin{array}{l}p=0.46 \\
t=0.82\end{array}$} & $\begin{array}{c}2.52 \pm \\
0.50\end{array}$ & \multirow{2}{*}{$\begin{array}{l}p=0.60 \\
t=0.57\end{array}$} & $\begin{array}{c}5.75 \pm \\
0.70\end{array}$ & \multirow{2}{*}{$\begin{array}{l}p=0.12 \\
t=2.13\end{array}$} & $\begin{array}{c}6.80 \pm \\
0.80\end{array}$ & \multirow{2}{*}{$\begin{aligned} p & =0.33 \\
t & =1.14\end{aligned}$} \\
\hline & $\begin{array}{l}\text { Female } \\
(n=4)\end{array}$ & $\begin{array}{c}0.81 \pm \\
0.08\end{array}$ & & $\begin{array}{c}2.25 \pm \\
0.23\end{array}$ & & $\begin{array}{c}2.35 \pm \\
0.70\end{array}$ & & $\begin{array}{c}6.90 \pm \\
0.70\end{array}$ & & $\begin{array}{c}7.40 \pm \\
0.30\end{array}$ & \\
\hline \multirow[t]{2}{*}{ NAC } & $\begin{array}{l}\text { Male } \\
(n=4)\end{array}$ & $\begin{array}{c}1.75 \pm \\
0.60\end{array}$ & \multirow{2}{*}{$\begin{aligned} p & =0.90 \\
t & =0.12\end{aligned}$} & $\begin{array}{c}1.65 \pm \\
0.19\end{array}$ & \multirow{2}{*}{$\begin{array}{l}p=0.54 \\
t=0.67\end{array}$} & $\begin{array}{c}2.52 \pm \\
0.90\end{array}$ & \multirow{2}{*}{$\begin{aligned} p & =0.60 \\
t & =0.57\end{aligned}$} & $\begin{array}{l}5.81 \pm \\
0.90\end{array}$ & $p=0.29$ & $\begin{array}{l}7.37 \pm \\
0.40\end{array}$ & \multirow{2}{*}{$\begin{array}{l}p=0.80 \\
t=0.14\end{array}$} \\
\hline & $\begin{array}{l}\text { Female } \\
(n=4)\end{array}$ & $\begin{array}{c}1.82 \pm \\
0.80 \\
\end{array}$ & & $\begin{array}{c}1.99 \pm \\
0.35\end{array}$ & & $\begin{array}{c}2.11 \pm \\
0.90 \\
\end{array}$ & & $\begin{array}{c}7.29 \pm \\
1.50 \\
\end{array}$ & $t=1.27$ & $\begin{array}{c}7.25 \pm \\
1.40 \\
\end{array}$ & \\
\hline \multirow[t]{2}{*}{ EGS } & $\begin{array}{l}\text { Male } \\
(n=4)\end{array}$ & $\begin{array}{c}0.72 \pm \\
0.30\end{array}$ & \multirow{2}{*}{$\begin{array}{l}p=0.31 \\
t=1.21\end{array}$} & $\begin{array}{c}0.90 \pm \\
0.59 \\
\end{array}$ & \multirow{2}{*}{$\begin{array}{l}p=0.96 \\
t=0.53\end{array}$} & $\begin{array}{c}1.29 \pm \\
0.72 \\
\end{array}$ & \multirow{2}{*}{$\begin{array}{l}p=0.94 \\
t=0.07\end{array}$} & $\begin{array}{c}3.85 \pm \\
0.70 \\
\end{array}$ & $p=0.71$ & $\begin{array}{c}8.70 \pm \\
1.01\end{array}$ & \multirow{2}{*}{$\begin{array}{l}p=0.58 \\
t=0.61\end{array}$} \\
\hline & $\begin{array}{l}\text { Female } \\
(n=4)\end{array}$ & $\begin{array}{c}0.60 \pm \\
0.10\end{array}$ & & $\begin{array}{c}0.87 \pm \\
0.57\end{array}$ & & $\begin{array}{c}1.34 \pm \\
0.60\end{array}$ & & $\begin{array}{c}4.21 \pm \\
1.50\end{array}$ & $t=0.40$ & $\begin{array}{c}9.15 \pm \\
1.10\end{array}$ & \\
\hline \multirow[t]{2}{*}{ LGS } & $\begin{array}{l}\text { Male } \\
(n=4)\end{array}$ & $\begin{array}{l}0.85 \pm \\
0.40\end{array}$ & \multirow{2}{*}{$\begin{array}{l}p=0.62 \\
t=0.54\end{array}$} & $\begin{array}{c}0.45 \pm \\
0.69\end{array}$ & \multirow{2}{*}{$\begin{array}{l}p=0.48 \\
t=0.80\end{array}$} & $\begin{array}{l}0.96 \pm \\
0.25\end{array}$ & \multirow{2}{*}{$\begin{aligned} p & =0.91 \\
t & =0.11\end{aligned}$} & $\begin{array}{c}4.45 \pm \\
0.70\end{array}$ & $p=0.43$ & $\begin{array}{c}8.25 \pm \\
1.18\end{array}$ & \multirow{2}{*}{$\begin{aligned} p & =0.06 \\
t & =2.77\end{aligned}$} \\
\hline & $\begin{array}{l}\text { Female } \\
(n=4)\end{array}$ & $\begin{array}{l}0.62 \pm \\
0.40\end{array}$ & & $\begin{array}{c}0.83 \pm \\
0.56\end{array}$ & & $\begin{array}{c}1.39 \pm \\
0.49\end{array}$ & & $\begin{array}{c}3.96 \pm \\
0.15\end{array}$ & $t=0.91$ & $\begin{array}{c}10.50 \pm \\
1.30\end{array}$ & \\
\hline \multirow{2}{*}{$\begin{array}{l}\text { EGS+ } \\
\text { NAC }\end{array}$} & $\begin{array}{l}\text { Male } \\
(n=4)\end{array}$ & $\begin{array}{c}1.70 \pm \\
0.70\end{array}$ & \multirow{2}{*}{$\begin{array}{l}p=0.82 \\
t=0.24\end{array}$} & $\begin{array}{c}2.25 \pm \\
1.10\end{array}$ & \multirow{2}{*}{$\begin{array}{l}p=0.30 \\
t=1.00\end{array}$} & $\begin{array}{c}2.58 \pm \\
0.57\end{array}$ & \multirow{2}{*}{$\begin{array}{l}p=0.62 \\
t=0.54\end{array}$} & $\begin{array}{c}5.88 \pm \\
2.10\end{array}$ & $p=0.71$ & $\begin{array}{c}7.27 \pm \\
0.70\end{array}$ & \multirow{2}{*}{$\begin{array}{l}p=0.66 \\
t=0.47\end{array}$} \\
\hline & $\begin{array}{l}\text { Female } \\
(n=4)\end{array}$ & $\begin{array}{c}1.80 \pm \\
0.80 \\
\end{array}$ & & $\begin{array}{c}1.67 \pm \\
0.23 \\
\end{array}$ & & $\begin{array}{c}2.30 \pm \\
0.61 \\
\end{array}$ & & $\begin{array}{c}6.19 \pm \\
0.90\end{array}$ & & $\begin{array}{c}7.05 \pm \\
0.20\end{array}$ & \\
\hline \multirow{2}{*}{$\begin{array}{l}\text { LGS+ } \\
\text { NAC }\end{array}$} & $\begin{array}{l}\text { Male } \\
(n=4)\end{array}$ & $\begin{array}{c}1.82 \pm \\
1.21\end{array}$ & \multirow{2}{*}{$\begin{array}{l}p=0.85 \\
t=0.19\end{array}$} & $\begin{array}{c}1.70 \pm \\
0.70\end{array}$ & \multirow{2}{*}{$\begin{array}{l}p=0.39 \\
t=0.98\end{array}$} & $\begin{array}{c}2.68 \pm \\
0.70\end{array}$ & \multirow{2}{*}{$\begin{aligned} p & =0.28 \\
t & =1.29\end{aligned}$} & $\begin{array}{c}5.00 \pm \\
0.60\end{array}$ & & $\begin{array}{c}7.45 \pm \\
0.90\end{array}$ & $p=0.48$ \\
\hline & $\begin{array}{l}\text { Female } \\
(n=4)\end{array}$ & $\begin{array}{c}1.95 \pm \\
0.88\end{array}$ & & $\begin{array}{c}2.20 \pm \\
0.50\end{array}$ & & $\begin{array}{c}2.01 \pm \\
0.40\end{array}$ & & $\begin{array}{l}7.50 \pm \\
1.00\end{array}$ & $t=3.07$ & $\begin{array}{c}7.70 \pm \\
1.27\end{array}$ & $t=0.80$ \\
\hline
\end{tabular}


Table I. Cont.

\begin{tabular}{|c|c|c|c|c|c|c|c|c|c|c|c|}
\hline \multicolumn{12}{|c|}{ Brain oxidant/antioxidants (PND 48) } \\
\hline & & \multicolumn{2}{|c|}{$\begin{array}{l}\text { Total antioxidants } \\
(\mathrm{mmol} / \mathrm{l})\end{array}$} & \multicolumn{2}{|c|}{$\begin{array}{c}\text { Superoxide } \\
\text { dismutase }(\mathrm{U} / \mathrm{mg} \\
\text { protein) }\end{array}$} & \multicolumn{2}{|c|}{$\begin{array}{c}\text { Reduced } \\
\text { glutathione (mg/ } \\
\text { gm brain tissue) }\end{array}$} & \multicolumn{2}{|c|}{$\begin{array}{c}\text { Glutathione reductase } \\
\text { (nmol NADPH oxidized/ } \\
\text { min/mg protein) }\end{array}$} & \multicolumn{2}{|c|}{$\begin{array}{l}\text { Lipid } \\
\text { peroxidation } \\
(\mathrm{ng} / \mathrm{gm})\end{array}$} \\
\hline \multirow[t]{2}{*}{ CON } & $\begin{array}{c}\text { Male } \\
(n=4)\end{array}$ & $\begin{array}{c}2.95 \pm \\
0.80\end{array}$ & \multirow{2}{*}{$\begin{array}{l}p=0.46 \\
t=0.82\end{array}$} & $\begin{array}{c}3.80 \pm \\
1.10\end{array}$ & \multirow{2}{*}{$\begin{array}{l}p=0.81 \\
t=0.24\end{array}$} & $\begin{array}{c}3.87 \pm \\
0.70\end{array}$ & \multirow{2}{*}{$\begin{array}{l}p=0.20 \\
t=1.60\end{array}$} & $\begin{array}{c}7.05 \pm \\
1.70\end{array}$ & \multirow{2}{*}{$\begin{array}{l}p=0.64 \\
t=0.50\end{array}$} & $\begin{array}{c}4.07 \pm \\
1.00\end{array}$ & \multirow{2}{*}{$\begin{array}{l}p=0.45 \\
t=0.86\end{array}$} \\
\hline & $\begin{array}{l}\text { Female } \\
(n=4)\end{array}$ & $\begin{array}{c}2.52 \pm \\
0.70\end{array}$ & & $\begin{array}{c}3.60 \pm \\
1.80\end{array}$ & & $\begin{array}{c}2.92 \pm \\
0.40\end{array}$ & & $\begin{array}{c}6.49 \pm \\
1.40\end{array}$ & & $\begin{array}{c}4.92 \pm \\
2.10\end{array}$ & \\
\hline \multirow[t]{2}{*}{ NAC } & $\begin{array}{c}\text { Male } \\
(n=4)\end{array}$ & $\begin{array}{c}2.28 \pm \\
1.70\end{array}$ & \multirow{2}{*}{$\begin{aligned} p & =0.06 \\
t & =1.40\end{aligned}$} & $\begin{array}{c}2.67 \pm \\
0.50\end{array}$ & \multirow{2}{*}{$\begin{array}{l}p=0.21 \\
t=1.56\end{array}$} & $\begin{array}{c}2.65 \pm \\
0.40\end{array}$ & \multirow{2}{*}{$\begin{aligned} p & =0.25 \\
t & =1.40\end{aligned}$} & $\begin{array}{c}7.78 \pm \\
2.80\end{array}$ & \multirow{2}{*}{$\begin{array}{l}p=0.47 \\
t=0.82\end{array}$} & $\begin{array}{c}4.80 \pm \\
1.30\end{array}$ & \multirow{2}{*}{$\begin{aligned} p & =0.64 \\
t & =0.51\end{aligned}$} \\
\hline & $\begin{array}{l}\text { Female } \\
(n=4)\end{array}$ & $\begin{array}{c}2.82 \pm \\
1.40\end{array}$ & & $\begin{array}{c}4.36 \pm \\
1.60\end{array}$ & & $\begin{array}{c}3.20 \pm \\
0.90\end{array}$ & & $\begin{array}{c}6.06 \pm \\
2.00\end{array}$ & & $\begin{array}{c}4.25 \pm \\
1.05\end{array}$ & \\
\hline \multirow[t]{2}{*}{ EGS } & $\begin{array}{c}\text { Male } \\
(n=4)\end{array}$ & $\begin{array}{c}2.48 \pm \\
1.30\end{array}$ & \multirow{2}{*}{$\begin{aligned} p & =0.27 \\
t & =1.32\end{aligned}$} & $\begin{array}{c}3.00 \pm \\
1.71\end{array}$ & \multirow{2}{*}{$\begin{aligned} p & =0.91 \\
t & =0.11\end{aligned}$} & $\begin{array}{c}2.36 \pm \\
0.80\end{array}$ & \multirow{2}{*}{$\begin{array}{l}p=0.54 \\
t=0.68\end{array}$} & $\begin{array}{c}4.70 \pm \\
1.80\end{array}$ & \multirow{2}{*}{$\begin{array}{l}p=0.82 \\
t=0.24\end{array}$} & $\begin{array}{c}6.72 \pm \\
1.20\end{array}$ & \multirow{2}{*}{$\begin{array}{l}p=0.98 \\
t=0.02\end{array}$} \\
\hline & $\begin{array}{l}\text { Female } \\
(n=4)\end{array}$ & $\begin{array}{c}1.50 \pm \\
0.90\end{array}$ & & $\begin{array}{c}3.08 \pm \\
2.24\end{array}$ & & $\begin{array}{c}2.83 \pm \\
0.90\end{array}$ & & $\begin{array}{c}5.00 \pm \\
0.90\end{array}$ & & $\begin{array}{c}6.75 \pm \\
1.20\end{array}$ & \\
\hline \multirow[t]{2}{*}{ LGS } & $\begin{array}{l}\text { Male } \\
(n=4)\end{array}$ & $\begin{array}{c}1.47 \pm \\
1.00\end{array}$ & \multirow{2}{*}{$\begin{array}{l}p=0.50 \\
t=0.74\end{array}$} & $\begin{array}{c}3.02 \pm \\
2.08\end{array}$ & \multirow{2}{*}{$\begin{array}{l}p=0.35 \\
t=1.10\end{array}$} & $\begin{array}{c}2.97 \pm \\
0.54\end{array}$ & \multirow{2}{*}{$\begin{array}{l}p=0.96 \\
t=0.04\end{array}$} & $\begin{array}{c}6.06 \pm \\
2.50\end{array}$ & \multirow{2}{*}{$\begin{aligned} p & =0.24 \\
t & =1.42\end{aligned}$} & $\begin{array}{c}7.10 \pm \\
1.90\end{array}$ & \multirow{2}{*}{$\begin{aligned} p & =0.52 \\
t & =2.71\end{aligned}$} \\
\hline & $\begin{array}{l}\text { Female } \\
(n=4)\end{array}$ & $\begin{array}{c}1.90 \pm \\
0.50 \\
\end{array}$ & & $\begin{array}{c}1.96 \pm \\
0.30\end{array}$ & & $\begin{array}{c}2.99 \pm \\
0.84\end{array}$ & & $\begin{array}{c}3.70 \pm \\
0.70\end{array}$ & & $\begin{array}{c}6.40 \pm \\
0.50\end{array}$ & \\
\hline \multirow[t]{2}{*}{$E G S+N A C$} & $\begin{array}{c}\text { Male } \\
(n=4)\end{array}$ & $\begin{array}{c}1.90 \pm \\
0.50\end{array}$ & \multirow{2}{*}{$\begin{aligned} p & =0.28 \\
t & =1.31\end{aligned}$} & $\begin{array}{c}3.67 \pm \\
2.00\end{array}$ & \multirow{2}{*}{$\begin{aligned} p & =0.88 \\
t & =0.15\end{aligned}$} & $\begin{array}{c}3.08 \pm \\
0.93 \\
\end{array}$ & \multirow{2}{*}{$\begin{array}{l}p=0.55 \\
t=0.67\end{array}$} & $\begin{array}{c}7.15 \pm \\
2.40\end{array}$ & \multirow{2}{*}{$\begin{array}{l}p=0.49 \\
t=0.76\end{array}$} & $\begin{array}{c}4.25 \pm \\
1.60\end{array}$ & \multirow{2}{*}{$\begin{array}{l}p=0.47 \\
t=0.80\end{array}$} \\
\hline & $\begin{array}{c}\text { Female } \\
(n=4)\end{array}$ & $\begin{array}{c}2.14 \pm \\
1.51 \\
\end{array}$ & & $\begin{array}{c}3.59 \pm \\
1.40\end{array}$ & & $\begin{array}{c}2.70 \pm \\
0.34\end{array}$ & & $\begin{array}{c}6.16 \pm \\
1.60\end{array}$ & & $\begin{array}{c}4.92 \pm \\
0.90\end{array}$ & \\
\hline LGS+NAC & $\begin{array}{l}\text { Male } \\
(n=4)\end{array}$ & $\begin{array}{c}3.35 \pm \\
1.70\end{array}$ & $p=0.25$ & $\begin{array}{c}3.50 \pm \\
0.60\end{array}$ & $p=0.90$ & $\begin{array}{c}3.31 \pm \\
0.70\end{array}$ & $p=0.68$ & $\begin{array}{c}4.20 \pm \\
1.14\end{array}$ & $p=0.67$ & $\begin{array}{c}4.20 \pm \\
1.10\end{array}$ & $p=0.67$ \\
\hline & $\begin{array}{l}\text { Female } \\
(n=4)\end{array}$ & $\begin{array}{c}2.32 \pm \\
1.10\end{array}$ & & $\begin{array}{c}3.40 \pm \\
0.60\end{array}$ & & $\begin{array}{c}3.12 \pm \\
0.92\end{array}$ & $t=0.45$ & $\begin{array}{c}4.80 \pm \\
1.08\end{array}$ & & $\begin{array}{c}4.80 \pm \\
1.80\end{array}$ & \\
\hline
\end{tabular}

$0.1 \mathrm{ml}$ of $10 \%$ trichloroacetic acid. After centrifugation $(1 \mathrm{ml})$ supernatant was then mixed with $1 \mathrm{ml}$ of $0.65 \%$ thiobarbituric acid. The mixture was then kept in a boiling water bath for $15 \mathrm{~min}$. The malondialdehyde formation was determined by reading absorbance at $535 \mathrm{~nm}$. The amount of TBARS was calculated using a molar extinction coefficient of $1.56 \times 10^{5}(\mathrm{M})^{-1} \mathrm{~cm}^{-1}$.

\section{Statistical analysis}

All results represent mean \pm S.E.M. The significance of differences among the groups was assessed using the one way analysis of variance (ANOVA) test followed by Bonferroni's multiple comparison test. Comparison of data between male and female groups was assessed by unpaired $t$ test. $P$ values $<0.05$ were considered significant.

\section{Results}

Brain antioxidant levels: There was no sexually dimorphic effect in all the parameters studied, hence mean values for both male and female rats were combined.

\section{Prenatal stress-induced declined total antioxidant capacity (TAO) is reversed by NAC}

\section{Effect of prenatal stress and NAC on total antioxidant capacity at PND 24}

A significant $(p<0.05)$ decrease in TAO was observed in the early gestational stress group when compared to the control group. Pups exposed to early gestational stress, treated with NAC (group 5) showed an increase $(p<0.05)$ in TAO when compared to the group that received early gestational stress alone. Total antioxidant capacity significantly decreased in group $4(p<0.05)$ whereas in group 6 , there was a significant elevation of TAO in brain homogenate. There was no significant difference in the level of TAO in the group that received only NAC when compared to the control group (Fig. 1). 
Effect of prenatal stress and NAC on total antioxidant capacity at PND 48

Total antioxidant capacity levels did not significantly differ between any of the comparisons (Fig. 1).

\section{Comparison of total antioxidant capacity between PND24 and PND48}

Total antioxidant capacity was seen to have significantly increased in group $3(p<0.01)$ and group 4 $(p<0.01)$, but did not show any elevation in group 1, 2, 5 and 6 at PND 48 when compared to PND 24.

\section{Prenatal stress-induced declined SOD activity is reversed by NAC}

Effect of prenatal stress and NAC on concentration of SOD at postnatal day 24 (PND 24)

There was a significant decrease $(p<0.05)$ in the level of SOD in offspring who received early ges-

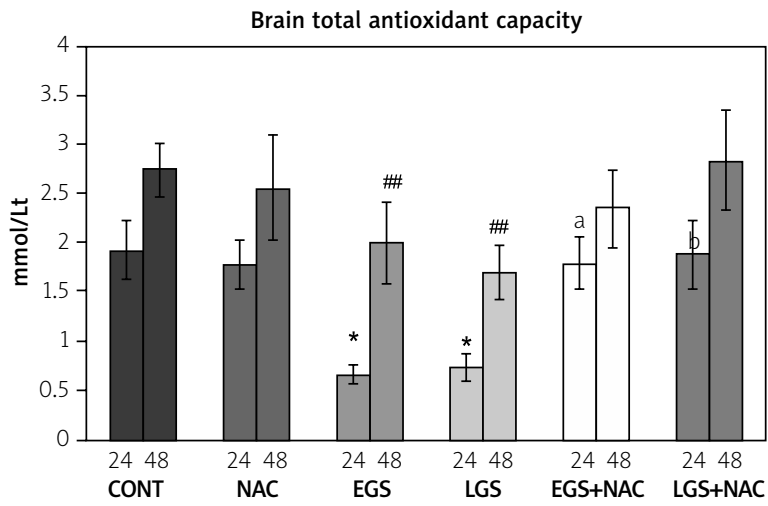

Fig. 1. Effect of prenatal stress and $\mathrm{N}$-acetyl cysteine (NAC) on brain TAO capacity in 24- and 48-day-old offspring. Abbreviations - CONT: control, EGS: rats received prenatal stress from gestation day 1 to 10, EGS+NAC: rats received stress from gestation day 1 to 10 along with $N$ acetyl cysteine during the entire gestation period, LGS: rats received prenatal stress from gestation day 11 until delivery, LGS+NAC: rats received stress from gestation day 11 to delivery along with $N$ acetyl cysteine during the entire gestation period, NAC: rats received $N$ acetyl cysteine for the entire gestation period. One way ANOVA, Bonferroni's test - CON vs. EGS\& LGS, ${ }^{*} p<0.05$; EGS vs. EGS+NAC, ${ }^{a} p<0.05$; LGS vs. LGS+NAC, ${ }^{b} p<0.05$; PND 24 vs. PND 48 , $\# \#<0.01$. tational stress (group 3) and also late gestational stress $(p<0.01)$ but group $5(p<0.05)$ and group 6 $(p<0.01)$ pups showed significantly elevated SOD levels when compared with those of group 3 and group 4, respectively. No significant difference was seen in the SOD level in the group that received NAC alone when compared to control animals (Fig. 2).

\section{Effect of prenatal stress and NAC on concentration of SOD at PND 48}

Superoxide dismutase activity was not seen to differ significantly between any of the groups (Fig. 2).

\section{Comparison of the SOD level between PND24 and PND48}

There was significant increase in brain SOD levels in all groups at PND 48 (group 1 [ $<<0.01$ ], group $2[p<0.05]$, group 3 [ $p<0.01$ ], group 4 [ $p<0.01]$, group $5[p<0.01])$ with respect to its levels at postnatal day 24 .

\section{Prenatal stress-induced declined reduced GSH activity is reversed by NAC}

Effect of prenatal stress and NAC on concentration of reduced glutathione at PND 24

Prenatal stress during early gestation (group 3 ) caused a significant $(p<0.05)$ depletion of the reduc-

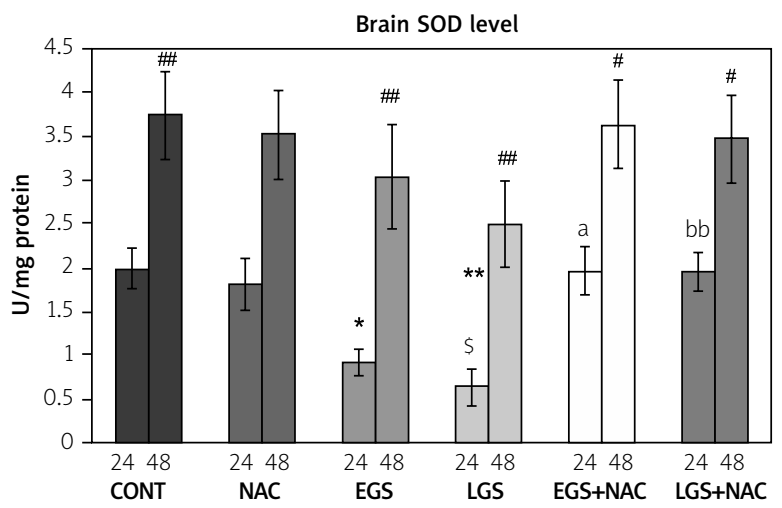

Fig. 2. Effect of prenatal stress and $\mathrm{N}$-acetyl cysteine (NAC) on brain SOD activity in 24- and 48-day-old offspring. Abbreviations - see Fig. 1. One way ANOVA, Bonferroni's test - CON vs. EGS $\&$ LGS, ${ }^{*} p<0.05,{ }^{* *} p<0.01$; EGS vs. EGS+NAC, ${ }^{a} p<0.05$; LGS vs. LGS+NAC, ${ }^{b b} p<0.01$; NAC vs. LGS, ${ }^{\$} p<0.05$; PND 24 vs. PND 48, ${ }^{\#} p<0.05$, $\# \#<0.01$. 
ed glutathione (GSH) level in the brain compared to controls (group 1). Offspring who received early gestational stress and NAC treatment (group 5) showed a significant $(p<0.05)$ increase in the GSH level when compared to offspring of group 3 . There was a significant $(p<0.01$ ) depletion of GSH concentration in the LGS group but offspring who received late gestational stress and NAC treatment (group 6) showed a significant $(p<0.01)$ increase in the GSH level when compared to offspring who received late prenatal stress. There was no significant difference in the level of GSH in the group that received only NAC when compared to the control group (Fig. 3).

\section{Effect of prenatal stress and NAC on concentration of reduced glutathione at postnatal day 48 (PND 48)}

The activity of brain GSSG-Rd did not show a statistically significant difference between any of the groups (Fig. 3).

\section{Comparison of a reduced glutathione concentration between PND24 and PND48}

Glutathione levels were significantly increased in group $1(p<0.05)$, group $3(p<0.01)$, group $4(p<0.001)$ and group $6(p<0.05)$ at postnatal day 48 in comparison with GSH levels at PND 24.

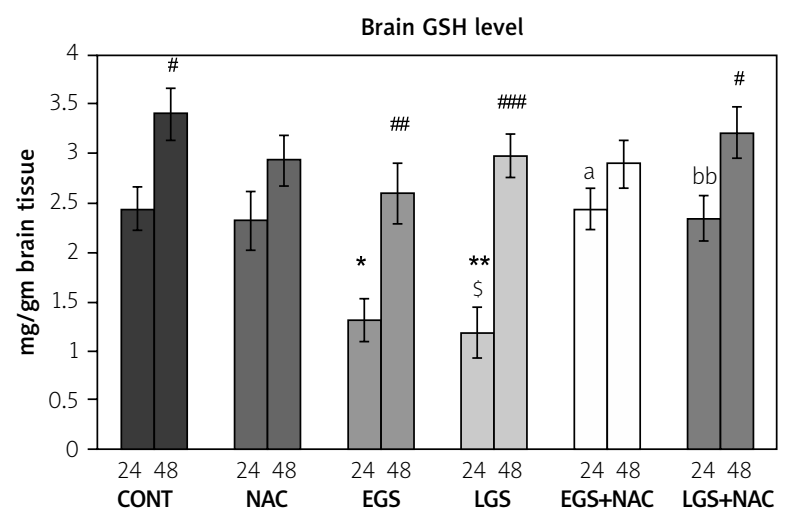

Fig. 3. Effect of prenatal stress and $N$-acetyl cysteine (NAC) on the brain reduced glutathione level in 24- and 48-day-old offspring. Abbreviations - see Fig. 1. One way ANOVA, Tukey-Kramer Multiple Comparisons Test - CON vs. EGS \& LGS, ${ }^{*} p<0.05$, ${ }^{* *} p<0.01$; EGS vs. EGS+NAC, ${ }^{a} p<0.05$; LGS vs. LGS+NAC, ${ }^{b b} p<0.01$; NAC vs. LGS, ${ }^{\$} p<0.05 ;$ PND 48 vs. PND 24, ${ }^{\#} p<0.05,{ }^{\# \#} p<0.01,{ }^{\# \# \# p<0.001 .}$

\section{Prenatal stress-induced decline in glutathione reductase (GSHRd) is reversed by NAC}

\section{Effect of prenatal stress and NAC on activity of glutathione reductase at PND 24}

The activity of glutathione reductase in the brain homogenate of different groups is summarized in Figure 4. GSHRd was significantly $(p<0.05)$ reduced in the offspring that belonged to the early gestational stress group when compared to controls. EGS offspring treated with prenatal NAC showed an elevation in GSSG-Rd, which was quite significant ( $p<0.05$ ) when compared to the early gestational stress group. It was seen that late gestational stress also significantly $(p<0.05)$ lowered the activity of reduced glutathione in comparison with control pups, whereas this activity was restored in the LGS+NAC group. No significant difference was seen in the GSHRd level in the group that received NAC alone when compared to control animals.

\section{Effect of prenatal stress and NAC on activity of glutathione reductase at PND 48}

The activity of brain GSSG-Rd did not show a statistically significant difference between any of the groups (Fig. 4).

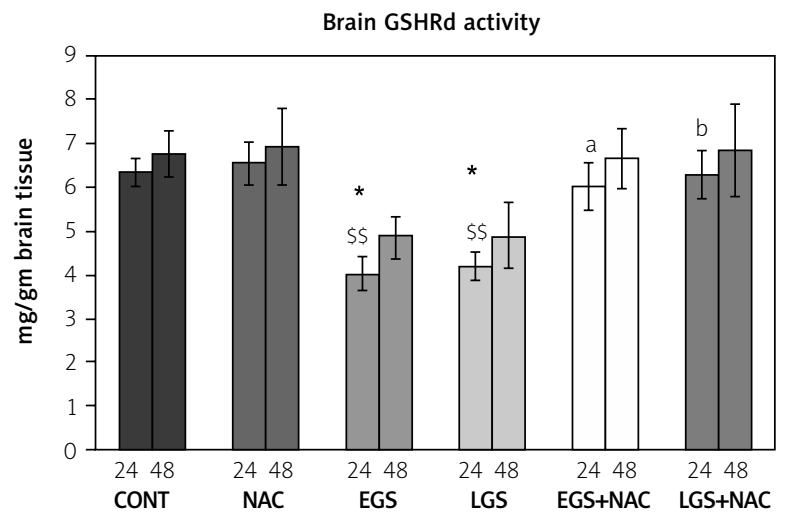

Fig. 4. Effect of prenatal stress and $N$-acetyl cysteine (NAC) on brain glutathione reductase activity in 24- and 48-day-old offspring. Abbreviations - see Fig. 1. One way ANOVA, Tukey-Kramer Multiple Comparisons Test - CON vs. EGS \& LGS, ${ }^{*} p<0.05$; EGS vs. EGS+NAC, ${ }^{a} p<0.05$; LGS vs. LGS+NAC, ${ }^{b} p<0.05$; NAC vs. EGS\&LGS, $\$ p<0.05, \$ \$ p<0.01$ 


\section{Comparison of glutathione reductase activity between PND24 and PND48}

As shown in Figure 4, no significant change in brain GSH Rd activity was observed in any of the groups at PND 48, when compared to its levels at PND 24.

\section{Prenatal stress-induced elevated lipid peroxidation is reversed by NAC}

\section{Effect of prenatal stress and NAC on lipid} peroxidation at postnatal day 24 (PND 24)

The malondialdehyde (MDA) level is a marker of lipid peroxidation. Early gestational stress significantly $(p<0.05)$ increased brain lipid peroxidation in comparison with the control group. A significant $(p<0.05)$ reduction in lipid peroxidation was observed in the early gestational stress group which received NAC (group 5) when compared to group 3. Brain lipid peroxidation was also markedly $(p<0.001)$ increased in the late gestational stress group (group 4) when compared to that of the control group. There was a significant $(p<0.05)$ decrease in the MDA level in the LGS+NAC group as compared with the LGS group. There was no significant difference in the level of MDA in the group that received only NAC when compared to the control group (Fig. 5).

\section{Effect of prenatal stress and NAC on lipid peroxidation at postnatal day 48 (PND 48)}

As summarized in Figure 5 , a significant $(p<0.05)$ elevation of lipid peroxidation activity in the brain homogenate was observed in the EGS group in comparison with the control group. EGS+NAC showed a significant $(p<0.05)$ decrease in lipid peroxidation when compared to EGS. The MDA level was also elevated significantly $(p<0.05)$ in LGS, whereas in LGS+NAC, a significant $(p<0.05)$ depletion in the MDA level was observed.

\section{Comparison of lipid peroxidation between PND24 and PND48}

There was a very significant reduction in brain MDA levels measured at PND 48, in the control group (group 1) $(p<0.001)$, group $3(p<0.001)$, group 4 $(p<0.0001)$, group $5(p<0.04)$ and group $6(p<0.0002)$ when compared to MDA levels measured at PND 24.

\section{Discussion}

Oxidative stress has been recognized as one of the earliest factors causing neurodegeneration [3] as

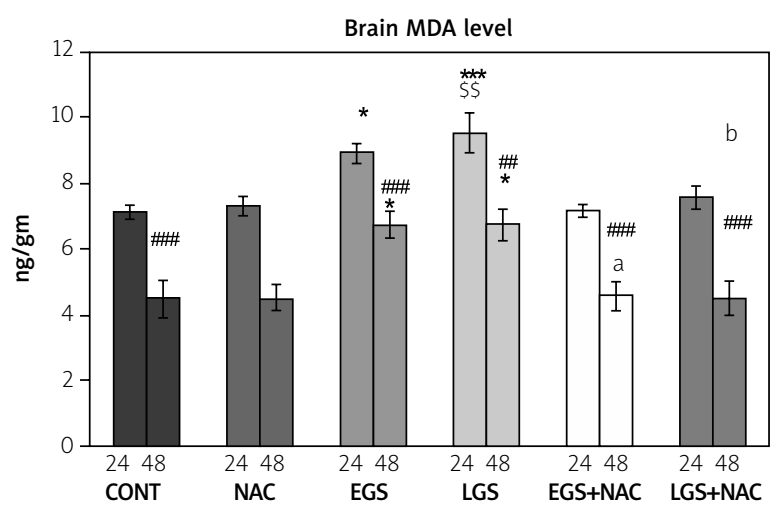

Fig. 5. Effect of prenatal stress and $\mathrm{N}$-acetyl cysteine (NAC) on brain lipid peroxidation (MDA level) in 24- and 48-day-old offspring. For all the parameters and legends, data are expressed as mean $\pm \mathrm{SEM}, n=8$, and animal groups are abbreviated as in Fig. 1. One way ANOVA, Tukey-Kramer Multiple Comparisons Test - CON vs. EGS\&LGS, ${ }^{*} p<0.05,{ }^{* * *} p<0.001$; EGS vs. EGS+NAC, ${ }^{a} p<0.05$; LGS vs. LGS+NAC, ${ }^{b} p<0.05$; NAC vs. LGS, $\$ \$ p<0.01$; PND 48 vs. PND 24, \#\# $p<0.01$, $\# \#$ \#\# 0.001.

brain is relatively more vulnerable to oxidative damage due to high levels of oxidizable polyunsaturated fatty acid and redox-active metals, but lower levels of antioxidant molecules [9]. It has previously been shown that oxidative damage produced by prenatal restrain stress resulted in neuronal loss in rat offspring [22C]. Exposure to early as well as late gestational stress brought about brain oxidative damage where decline in SOD, GSH and GSSG-Rd activities $[23,39]$, increase in lipid peroxidation and decrease in total antioxidant capacity were observed [22]. An increase in the content of reactive oxygen species in the hippocampus of prenatally stressed rat offspring was reported by Li et al. [20]. In agreement with these studies, we found that early as well as late prenatal stress resulted in brain oxidative stress in 24-day-old offspring as indicated by a decrease in levels of total antioxidants, SOD, GSH and GSH-Rd, as well as an increase in lipid peroxidation. These findings reveal suppressed efficiency of antioxidants to combat the prenatal stress induced ROS in the developing brain.

Super oxide dismutases that form a very important component of antioxidant defenses are a class of metalloenzymes that catalyze dismutation of the superoxide radical into oxygen and $\mathrm{H}_{2} \mathrm{O}_{2}$ [13]. Our data indicated that prenatal stress has resulted in 
significant inhibition of SOD and glutathione reductase activities. This reduction could be a result of increased ROS generation and enhanced lipid peroxidation [5].

Glutathione is required for detoxification of ROS in brain cells [8]. The decrease in GSH concentrations we observed in this study could be due to its increased rate of utilization caused by oxidative stress [36]. Increased levels of free radicals bring about more reduced glutathione (GSH) to convert to its oxidized form (GSSG) [32]. It could also be due to a decreased activity of the enzyme glutathione reductase [6] that is required to restore the GSH level.

Lipid peroxidation is considered as a major source of oxidative damage by stress. The brain being rich in polyunsaturated lipids, and having an elevated rate of oxygen consumption, is highly susceptible to peroxidation by oxygen free radicals $[35,15]$. Malondialdehyde, a marker of lipid peroxidation, is very reactive and plays a role in cross-linking with DNA and proteins, resulting in mitochondrial dysfunction and cell damage [11]. In the present study, early as well as late prenatal stress has brought about a significant elevation in MDA levels, in 24-day-old offspring, as a possible consequence of decreased antioxidant enzyme activities.

The results of our study demonstrated that prenatal NAC treatment enhanced the activity of the antioxidant defense system in the offspring who received prenatal stress during early as well as late gestation. It prevented the decrease in GSH, GSSG$\mathrm{Rd}, \mathrm{SOD}$ and total antioxidant levels and increase in lipid peroxidation in the brain of prenatally stressed offspring, thus proving its neuroprotective role against oxidative damage.

In the present work, we also evaluated the levels of total antioxidant capacity, SOD, GSH, GSSG-Rd and MDA at PND 48 to investigate the long-term effects of early and late gestational stress on the brain antioxidant system; PND 48 in rats corresponds to the early adult period in human subjects. Interestingly, however not expected result suggestive of brain oxidative damage was detected except for a small but significant increase in MDA levels. This shows the possibility of recovery of the brain from gestational stress-induced oxidative damage when the rats reached the early adult stage. Not many studies, to the best of our knowledge, have investigated the long-term effect of gestational restrain stress on oxidative damage in rat whole brain homogenate, although several animal and human studies have proved long-term effects of different kinds of stress during pregnancy in terms of behavior, physiology, and immunity of the offspring $[41,42]$. In several studies where long-term effects of prenatal stress were investigated, younger offspring showed higher emotionality levels when compared to older ones [40], higher anxiety and lower locomotion at 1 month, but not at 4 months [2], decrease in dendritic spines in pyramidal neurons of offspring at PND 23 in a study by Murmu et al. [28], although no such decrease at PND 100 in response to a similar kind of a repeated prenatal stressor as reported by Michelson et al. [26]. In a study by Kraszpulski et al., the decrease in volumes and cell numbers in amygdala resolved after PND 45 [18]. Based on these data and the result of our present study, the response of the offspring brain to prenatal stress appears to at times be short lived and the brain seems to recover from this transient stress effect. Such variation in responses may occur depending on the type, duration, intensity of stress and different strains of animal species. We also compared the brain oxidative status of individual groups between 2 ages where we observed a significant increase in total antioxidant capacity, SOD, GSH, and fall in lipid peroxidation, in all groups at PND 48 when compared to their levels at PND 24. This shows that brain oxidative defense improves with age at early development periods. However oxidative stress during early postnatal development is sufficient enough to adversely affect the neurobehavioral development.

\section{Conclusions}

In summary, the present study demonstrated that both early and late gestational stress showed oxidative stress in the developing brain during early postnatal development and this effect did not last until postnatal day 48 , which suggests that oxidative stress may be transient and last for a short period of postnatal development. Our data suggest that maternal administration of NAC effectively prevented prenatal stress-induced oxidative changes in the developing brain of the offspring exposed to prenatal stress. Based on the results of this study, we assume that the NAC can be beneficial for preventing the brain oxidative damage and cognitive dysfunction associated with prenatal stress, hence an ideal candidate for clinical trials. 


\section{Disclosure}

\section{Authors report no conflict of interest.}

\section{References}

1. Basyigit I, Tugay M, Dilioglugil MO, Yildiz F, Maral H, Sozubir S. Protective effects of $\mathrm{N}$-acetylcysteine on peroxidative changes of the fetal rat lungs whose mothers were exposed to cigarette smoke. Hum Exp Toxicol 2007; 26: 99-103.

2. Batuev AS, Vinogradova EP, Polyakova ON. The effect of prenatal stress on rat offspring anxiety. Zh Vyssh Nerv Deiat Im I P Pavlova 1996; 46: 558-563.

3. Beal MF. Does impairment of energy metabolism result in excitotoxic neuronal death in neurodegenerative illnesses? Ann Neurol 1992; 31: 119-130.

4. Burton GJ, Jauniaux E. Oxidative stress. Best Pract Res Clin Obstet Gynaecol 2011; 25: 287-299.

5. Chaudiere J, Ferrari-lliou R. Intracellular antioxidants: from chemical to biochemical mechanisms. Food Chem Toxicol 1999; 37: 949-962

6. Costagliola C. Oxidative state of glutathione in red blood cells and plasma of diabetic patients: in vivo and in vitro study. Clin Physiol Biochem 1989; 8: 204-210.

7. Dringen R, Hamprecht B. N-acetylcysteine, but not methionine or 2-oxothiazolidine-4-carboxylate, serves as cysteine donor for the synthesis of glutathione in cultured neurons derived from embryonal rat brain. Neurosci Lett 1999; 259: 79-82.

8. Dringen R, Hirrlinger J. Glutathione pathways in the brain. Biol Chem 2003; 384: 505-516.

9. Driver AS, Kodavanti PRS, Mundy WR. Age-related changes in reactive oxygen species production in rat brain homogenates. Neurotoxicol Teratol 2000; 22: 175-181.

10. Ellman GL. Tissue sulfhydryl groups. Arch Biochem Biophys 1959; 82: 70-77.

11. Esterbauer H. Cytotoxicity and genotoxicity of lipid-oxidation products. Am J Clin Nutr 1993; 57: 779S-785S.

12. Farr SA, Poon HF, Dogrukol-Ak D, Drake J, Banks WA, Eyerman E, Butterfield DA, Morley JE. The antioxidants $\alpha$-lipoic acid and $\mathrm{N}$-acetylcysteine reverse memory impairment and brain oxidative stress in aged SAMP8 mice. J Neurochem 2003; 84: 1173-1183.

13. Fridovich I. Superoxide radical and superoxide dismutases. Annu Rev Biochem 1995; 64: 97-112.

14. Garnier Y, Coumans ABC, Jensen A, Hasaart THM, Berger R. Infection-related perinatal brain injury: the pathogenic role of impaired fetal cardiovascular control. I Soc Gynecol Investig 2003; 10: 450-459.

15. Halliwell B. Role of free radicals in the neurodegenerative diseases. Drugs Aging 2001; 18: 685-716.

16. Kawamura T, Chen J, Takahashi T, Ichitani Y, Nakahara D. Prenatal stress suppresses cell proliferation in the early developing brain. Neuroreport 2006; 17: 1515-1518.

17. Koracevic D, Koracevic G, Djordjevic V, Andrejevic S, Cosic V. Method for the measurement of antioxidant activity in human fluids. J Clin Pathol 2001; 54: 356-361.

18. Kraszpulski M, Dickerson PA, Salm AK. Prenatal stress affects the developmental trajectory of the rat amygdala. Stress 2006; 9: 85-95.
19. Lante F, Meunier J, Guiramand J, De Jesus Ferreira M-Cl, Cambonie G, Aimar R, Cohen-Solal C, Maurice T, Vignes M, Barbanel $\mathrm{Gr}$. Late $\mathrm{N}$-acetylcysteine treatment prevents the deficits induced in the offspring of dams exposed to an immune stress during gestation. Hippocampus 2008; 18: 602-609.

20. Li H, Li X, Jia N, Cai Q, Bai Z, Chen R, Song T, Zhu Z, Liu J. NF-kappa $B$ regulates prenatal stress-induced cognitive impairment in offspring rats. Behav Neurosci 2008; 122: 331-339.

21. Lowry OH, Rosebrough NJ, Farr AL, Randall RJ. Protein measurement with the Folin phenol reagent. J Biol Chem 1951; 193: 265 275.

22. Madhyastha S, Sahu SS, Rao G. Resveratrol for prenatal-stress-induced oxidative damage in growing brain and its consequences on survival of neurons. J Basic Clin Physiol Pharmacol 2014; 25: 63-72.

23. Madhyastha S, Sahu SS, Rao GM. Prenatal stress-induced cognitive impairment and neuronal oxidative stress and its amelioration by resveratrol in neonate rats. Res J Pharm Biol Chem Sci 2012; 3: 1387-1399.

24. Madhyastha SK, Prabhu LV, Nayak S, Rai R, Pai MM, Madhyastha PS. Effect of Prenatal stress and serotonin depletion on postnatal serotonin metabolism in Wistar rats. Iranian J Pharmacol Ther 2008; 7: 71-77.

25. Marklund S, Marklund G. Involvement of the superoxide anion radical in the autoxidation of pyrogallol and a convenient assay for superoxide dismutase. Eur J Biochem 1974; 47: 469-474.

26. Michelsen KA, van den Hove DILA, Schmitz C, Segers O, Prickaerts J, Steinbusch HWM. Prenatal stress and subsequent exposure to chronic mild stress influence dendritic spine density and morphology in the rat medial prefrontal cortex. BMC Neurosci 2007; 8: 107.

27. Moron MS, Depierre JW, Mannervik B. Levels of glutathione, glutathione reductase and glutathione- S-transferase activities in rat lung and liver. Biochim Biophys Acta 1979; 582: 67-78.

28. Murmu MS, Salomon S, Biala Y, Weinstock M, Braun K, Bock Jr. Changes of spine density and dendritic complexity in the prefrontal cortex in offspring of mothers exposed to stress during pregnancy. Eur J Neurosci 2006; 24: 1477-1487.

29. Nehru B, Kanwar S. N-acetylcysteine exposure on lead-induced lipid peroxidative damage and oxidative defense system in brain regions of rats. Biol Trace Elem Res 2004; 101: 257-264.

30. Novais ARB, Guiramand J, Cohen-Solal C, Crouzin N, de Jesus Ferreira MC, Vignes M, Barbanel G, Cambonie G. N-acetyl-cysteine prevents pyramidal cell disarray and reelin-immunoreactive neuron deficiency in CA3 after prenatal immune challenge in rats. Pediatr Res 2013; 73: 750-755.

31. Ohkawa H, Ohishi N, Yagi K. Assay for lipid peroxides in animal tissues by thiobarbituric acid reaction. Anal Biochem 1979; 95 : 351-358.

32. Ou P, Nourooz-Zadeh J, Tritschler H-J, Wolff S. Activation of aldose reductase in rat lens and metal-ion chelation by aldose reductase inhibitors and lipoic acid. Free Radic Res 1996; 25 : 337-346.

33. Paintlia MK, Paintlia AS, Barbosa E, Singh I, Singh AK. N-acetylcysteine prevents endotoxin-induced degeneration of oligodendrocyte progenitors and hypomyelination in developing rat brain. J Neurosci Res 2004; 78: 347-361. 
34. Rauchova H, Vokurkova M, Koudelova J. Hypoxia-induced lipid peroxidation in the brain during postnatal ontogenesis. Physiol Res 2012; 61: S89.

35. Rehncrona S, Mela L, Siesj BK. Recovery of brain mitochondrial function in the rat after complete and incomplete cerebral ischemia. Stroke 1979; 10: 437-446.

36. Sahin E, Gümüşlü S. Alterations in brain antioxidant status, protein oxidation and lipid peroxidation in response to different stress models. Behav Brain Res 2004; 155: 241-248.

37. Sastre J, Asensi M, Rodrigo F, Pallardo F, Vento M, Vina J. Antioxidant administration to the mother prevents oxidative stress associated with birth in the neonatal rat. Life Sci 1994; 54: 2055-2059.

38. Semple BD, Blomgren K, Gimlin K, Ferriero DM, Noble-Haeusslein LJ. Brain development in rodents and humans: Identifying benchmarks of maturation and vulnerability to injury across species. Prog Neurobiol 2013; 106: 1-16.

39. Sudhanshu Sahu SM, Gayathri Rao. Effect of prenatal stress on expression of glutathione system in neonatal rat brain. Turk Neurosurg 22: 576-582.

40. Takahashi LK, Turner JG, Kalin NH. Prenatal stress alters brain catecholaminergic activity and potentiates stress-induced behavior in adult rats. Brain Res 1992; 574: 131-137.

41. Talge NM, Neal C, Glover V. Antenatal maternal stress and long term effects on child neurodevelopment: how and why? J Child Psychol Psychiatry 2007; 48: 245-261.

42. Weinstock $M$. The long-term behavioural consequences of prenatal stress. Neurosci Biobehav Rev 2008; 32: 1073-1086.

43. Zhu Z, Li X, Chen W, Zhao Y, Li H, Qing C, Jia N, Bai Z, Liu J. Prenatal stress causes gender dependent neuronal loss and oxidative stress in rat hippocampus. J Neurosci Res 2004; 78 : 837-844.

44. Ziment I. Acetylcysteine: a drug that is much more than a mucokinetic. Biomed Pharmacother 1987; 42: 513-519. 